EPJ Web of Conferences 61, 03005 (2013)

DOI: $10.1051 /$ epjconf/ 20136103005

(C) Owned by the authors, published by EDP Sciences, 2013

\title{
Magnetic Field Structure in Relativistic Jets
}

\author{
Helen Jermak ${ }^{1, a}$, Carole Mundell ${ }^{1, b}$, lain Steele ${ }^{1, c}$, Richard Harrison ${ }^{1, d}$, Shiho Kobayashi ${ }^{1, e}$, Elina Lindfors ${ }^{2, f}$, Kari \\ Nilsson $^{2, g}$, and Ulisses Barres de Almeida ${ }^{3, h}$ \\ ${ }^{1}$ Astrophysics Research Institute, Liverpool John Moores University, IC2, Liverpool Science Park, Brownlow Hill, L3 5RF \\ ${ }^{2}$ Finnish Centre for Astronomy with ESO, University of Turku, Finland \\ ${ }^{3}$ Centro Brasileiro de Pesquisas Fisicas (CBPF/MCTI), Rua Dr. Xavier Sigaud 150, 22290-180 Rio de Janeiro RJ, Brazil
}

\begin{abstract}
Relativistic jets are ubiquitous when considering an accreting black hole. Two of the most extreme examples of these systems are blazars and gamma-ray bursts (GRBs), the jets of which are thought to be threaded with a magnetic field of unknown structure. The systems are made up of a black hole accreting matter and producing, as a result, relativistic jets of plasma from the poles of the black hole. Both systems are viewed as point sources from Earth, making it impossible to spatially resolve the jet. In order to explore the structure of the magnetic field within the jet we take polarisation measurements with the RINGO polarimeters on the world's largest fully autonomous, robotic optical telescope: The Liverpool Telescope. Using the polarisation degree and angle measured by the RINGO polarimeters it is possible to distinguish between global magnetic fields created in the central engine and random tangled magnetic fields produced locally in shocks. We also monitor blazar sources regularly during quiescence with periods of flaring monitored more intensively. Reported here are the early polarisation results for GRBs 060418 and 090102, along with future prospects for the Liverpool Telescope and the RINGO polarimeters.
\end{abstract}

\section{Introduction}

Gamma-ray bursts (GRBs) and blazars are the most extreme examples of black hole accretion systems in our visible Universe. Both systems have a black hole at the centre that accretes matter and as a result forms directionally opposed relativistic jets of plasma [1]. The sources differ in the size of central compact object. GRBs contain a stellar mass black hole and undergo a brief episode of hyperaccretion following one of two events: the collapse of a massive stellar object in the case of long-GRBs $\left(\mathrm{T}_{\gamma}>2\right.$ s) $[2,3]$ or possibly the merging of two compact objects (i.e. black hole-neutron star or neutron star-neutron star) for short GRBs $\left(\mathrm{T}_{\gamma}<2 \mathrm{~s}\right)$ [4]. Blazars are the more massive of the two systems. They are a sub-class of active galactic nuclei (AGN) which includes flat spectrum radio quasars (FSRQs) and BL Lacs. They contain a supermassive black hole $\left(\sim 10^{8}-10^{9} \mathrm{M}_{\odot}[5]\right)$ at their centre and undergo a longer period of accretion than GRBs: the matter under the gravitational influence of the black hole being continually drawn inwards [6]. The speeds of the different jets for these systems vary, blazars have Lorentz factors

\footnotetext{
a e-mail: h.e.jermak@ 2012.ljmu.ac.uk

be-mail: cgm@astro.livjm.ac.uk

ce-mail: i.a.steele@ljmu.ac.uk

d e-mail: r.m.harrison@2006.ljmu.ac.uk

e e-mail: s.kobayashi@ljmu.ac.uk

fe-mail: elilin@utu.fi

ge-mail: kani@utu.fi

he-mail: ulisses@cbpf.br
}

ranging from $\Gamma \sim 2-30$ [7] whilst GRB jets exhibit Lorentz factors ranging from $\Gamma \sim 100-1000$ [8]. Despite the differences in the size, speed and duration of these systems, the principle mechanism of jet formation through accretion onto a black hole is ubiquitous and makes the comparison of GRB and blazar jets a powerful tool in the study of jet physics [9].

The AGN unification system shows that the variety of observed quasar systems can be consolidated as the same AGN viewed from different angles: with a blazar being a subclass of AGN viewed within a small opening angle of the jet axis $([10,11])$. With increasing opening angle the system observed would be a core-dominated quasar, a lobe dominated quasar and finally, when the system is viewed side-on, a radio galaxy with both jets extending along the same plane $([10,11])$. It is possible that the same comparison can be made for systems with a stellar mass black hole at the centre. A GRB can be considered as analogous to a blazar as the system is viewed within a small opening angle of the jet. Although the true origin of these sources remains unknown, it is possible that with an increasing opening angle the X-ray rich GRBs (e.g. [12]) observed are analogous to core dominated quasars, and Xray flashes (e.g. [13]) are analogous with viewing the GRB side on (similar to a radio galaxy) [14].

Due to their orientation both GRBs and blazars are point-source objects which makes it impossible to spatially resolve the structure of the jet. For this reason we use polarimetric measurements to gain information about 
the magnetic field that threads through the jet. Blazars are easily monitored as they have a known location and are continually emitting sources. GRBs, however, are particularly difficult to study due to the fact they have short lifetime (early afterglow phase $\sim$ hours) and occur at unpredictable locations with magnitudes that are unknown prior to the outburst.

To be able to conduct regular and intensive monitoring of blazars and also probe the nature of GRB afterglows shortly after outburst, it is necessary to employ a robotic telescope that can respond to gamma-ray telescope triggers. A fully autonomous, robotic telescope such as the Liverpool Telescope is ideally designed for such use [15].

\section{The Liverpool Telescope}

The Liverpool Telescope (LT) is owned by the Astrophysics Research Institute of Liverpool John Moores University, UK. It was built in 2004 by Telescope Technologies Limited: a company linked with the university and is the world's largest fully autonomous robotic optical telescope [15]. The two-metre class telescope is located at the Roque de los Muchachos Observatory on the island of La Palma, Canary Islands and specialises in time variable and rapid reaction astronomy. The telescope has a clamshell design which gives it the advantage of an excellent view of the entire sky [15]. This fully open enclosure, combined with the telescope's fast slew time (2 degrees/second) makes it a powerful instrument for rapidly varying physics. The telescope is fully autonomous, it operates alone without human intervention and allows for intelligent dispatch scheduling, rather than queuing. The LT is ideally suited for the frequent monitoring and intensive observations of transient and variable sources such as GRBs and blazars. The LT has two clones: the Faulkes Telescope North (located at Haleakala Observatory, Hawaii, U.S.) and South (located at Siding Spring Observatory, New South Wales, Australia) both of which are used in addition to the LT by the Liverpool GRB group for the automated follow up of GRBs [16].

Since 2005, the LT has been responding to GRB triggers from the burst alert telescope (BAT) on the NASA SWIFT spacecraft [17]. Within seconds of the outburst occurring in gamma-rays, BAT produces a burst localisation which is sent to ground observers including the LT [18]. The LT initiates override mode and starts followup observations within 2-3 minutes of the GRB outburst. The initial short exposures are reduced and analysed using the LT-TRAP (Liverpool Telescope Transient Rapid Analysis Pipeline) software and if an unknown and potentially fading transient is recognised the telescope triggers a polarimetric imaging sequence using the polarimeter on the telescope [19].

We also use the LT to monitor a sample of gamma-ray selected AGN, predominantly blazars, which have gone into flaring mode since 2010. Along with its override mode to monitor GRB candidates, the telescope has a flare trigger mode which allows it to respond to triggers from the FERMI and MAGIC gamma-ray telescopes to monitor blazars when they are most active. There is also a program of regular monitoring to explore the behaviour of the sources when they are in quiescent mode.

\subsection{The RINGO polarimeters}

The LT is able to mount up to 9 instruments at the Cassegrain focus: one located at the 'straight through' position and eight more on side ports accessible by a rotating tertiary mirror [15]. One of these instruments is the optical polarimeter named RINGO (due to the ring projected onto the CCD by the eponymous instrument) [20]. RINGO was built in 2006 from a novel design [21], it was created to monitor the afterglow polarimetry of GRBs as soon after outburst as possible. The telescope is equipped with the LT-TRAP pipeline which responds to GRB triggers from the SWIFT satellite and produces automated early-time light curves of GRB afterglows ([22]). RINGO has a $>4 \times 4$ arcminute field of view and a rapidly rotating polaroid (550 rpm) which modulates the beam of polarised light entering the polarimeter from a particular source. This is followed by co-rotating deviating optics which record an image of the source onto a charge coupled device (CCD) chip in a ring pattern. The time-varying polarisation signal is mapped out in a $\sin (2 \theta)$ pattern around each recorded ring on the CCD [23]. The total intensity is then calculated by integrating around the annulus. Through using the equations specified in the Clarke \& Neumayer paper [21] the polarisation degree and angle can be calculated and used to compare to the theoretical predictions of the structure of the magnetic field shortly after outburst.

In 2009 RINGO was decommissioned and replaced by the second generation RINGO2 polarimeter. RINGO2 was designed to improve sensitivity by replacing the CCD from the original RINGO design with an electron multiplying charge coupled device (EMCCD) [23]. Without the need to convert the data into a spatial signal (i.e. the ring) the EMCCD can rapidly read out an image of the source at a rate of $125 \mathrm{msec}$ for each exposure. This reduces the excess sky signal and significantly improves the sensitivity [23]. RINGO2 was operational from 2009 until late 2012 when it was succeeded by RINGO3, the most current generation of the RINGO polarimeters.

RINGO3, the third generation polarimeter in the RINGO series, is a multicolour device fitted with dichroic mirrors that split the polarised incoming beam of light into three optical cameras (see Figure 1): blue-visible (350-640 $\mathrm{nm})$, green $(650-760 \mathrm{~nm})$ and red (770-1000 nm) simultaneously. This allows the measurement of polarisation degree and angle in three optical bands which can then be combined with a multicolour light curve (also simultaneous with the polarisation data) to help distinguish the particular area of the jet in which the polarisation signal occurs.

\section{Jet models}

The observed properties of GRBs and especially afterglows can be well described by the fireball model [24]. 


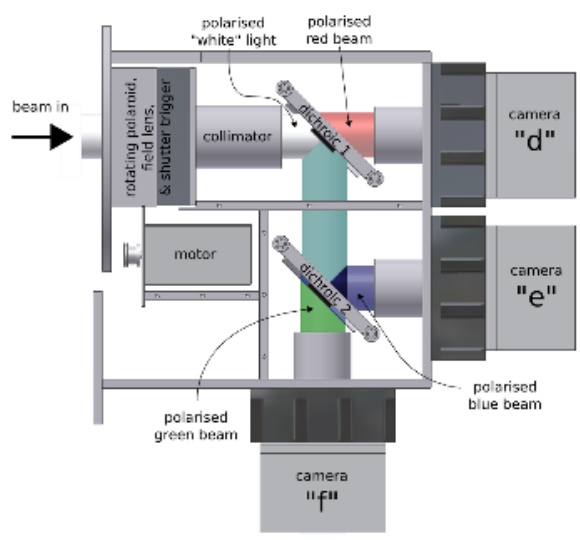

Figure 1. Schematic of the RINGO3 polarimeter showing the dichroic mirrors that split the incoming beam of polarised light to three separate cameras which simultaneously monitor the source in three wavelength bands [22].

The cause of the fireball explosion is believed to be the collapse of a massive stellar object (long GRB) [2,3] or the merger of two compact objects (short GRB) [4]. At the source of the explosion is the rapid accretion of matter onto a stellar mass black hole which launches a jet mode. This outflow can be thought of as discrete shells of material travelling at different velocities. The faster moving shells collide with the slower moving shells and create an internal shock within the jet (which emits gamma-rays) thus transferring a large amount of kinetic energy to the electrons which spiral around strong magnetic fields, producing synchrotron emission that can be up to $\sim 60-70 \%$ polarised [22]. When the jet plasma collides with the circumburst medium a forward and reverse shock are created the emission of which spans from radio to X-rays. The reverse shock propagates back into the jet (the original ejecta from the central engine) whilst the forward shock continues out into the surrounding medium. The forward shock region contains only the shocked ambient medium and it is insensitive to the magnetic structure in the original ejecta. The forward shock emission is expected to be unpolarised, while the reverse shock emission is highly polarised if the jet contains large-scale magnetic fields from the central engine.

The observed optical afterglow can theoretically have one of three light curve structures (see Figure 2), each curve shows the moment of deceleration of the fireball (the peak of the reverse shock emission) and the temporal progression of the forward and reverse shock emissions [25]. Theory predicts that the strength of the reverse shock emission depends on the magnetisation of the jet $([26,27])$ and in order to distinguish between the three light curves, the source needs to be observed and measurements taken as soon after the outburst as possible. With the autonomous capabilities and fast slew time of the LT it is possible to start observing the afterglow of a GRB in the first minutes after the outburst is detected in gamma-rays [15].

Figure 2 shows three theoretical lightcurves. In the Type I model the reverse and forward shock emissions are
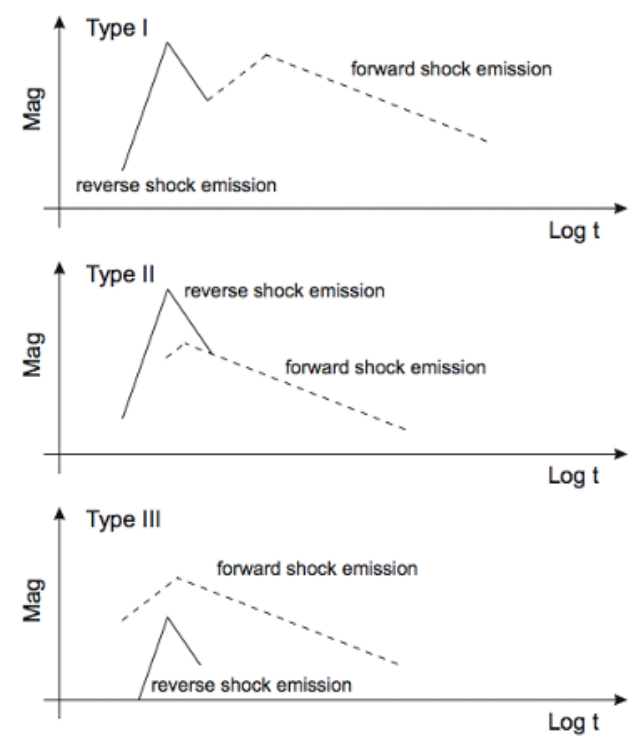

Figure 2. Three theoretical GRB light curves in which either the reverse or forward shock emissions dominate the curve. These theoretical predictions can be compared to observational light curves in order to explore the magnetisation of the jet [25].

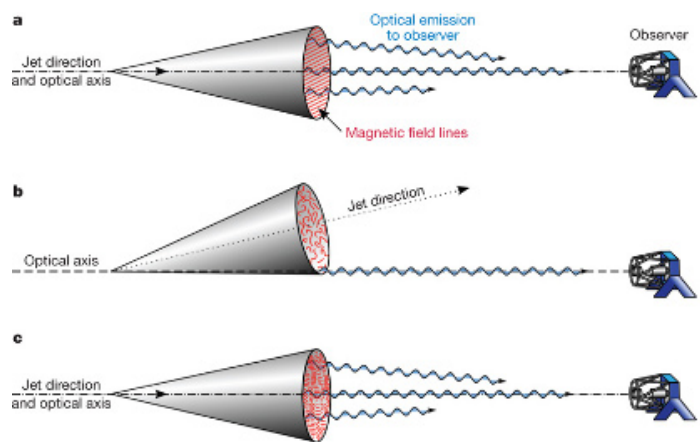

Figure 3. Three theoretical models of GRB jets in which a polarisation detection would be made. In the different models the magnetic field is ordered (a), disordered (b) and made up of coherent patches of ordered magnetic field (c) [25]. See text for full details.

both visible and the deceleration of the fireball is distinguishable. In the Type II model the deceleration of the fireball is distinguishable and the forward shock emission only becomes visible when the reverse shock has subsided. In the Type III model the forward shock dominates the emission and the reverse shock, along with the deceleration of the fireball, is not seen [25]. We require colour information around the peak to distinguish whether its origin lies in the fireball deceleration. The theoretical prediction that the forward shock would have low or no polarisation and the reverse shock would be strongly polarised is useful to confirm which of the models best describes the observed light curve.

The two main jet models can be summarised as hydrodynamical (no dominant ordered magnetic field and produce synchrotron radiation from tangled magnetic fields 
and shock instabilities [28]) and magnetised (jets threaded with strong, ordered magnetic fields originating at the central source [29, 30]) [33]. Figure 3 shows the three main theoretical models for the production of polarised light from GRB jets: large-scale ordered magnetic field (model a), random magnetic field (model b) and coherent patches of magnetic field (model c). Each of these jet models produces characteristic polarisation degree and angle evolution and can be used in comparison to observations to constrain the structure of the magnetic field within the jet.

Model a (see Figure 3) is a magnetised model showing the reverse shock emission of a GRB occurs within the large-scale ordered magnetic field of the outflow (e.g. [31]). This source is viewed within a small opening angle of the jet and the model predicts measurements of up to $70 \%$ polarisation, which decreases with time as the reverse shock emission declines and the unpolarised forward shock emission increases. The polarisation angle predicted by the model would be constant as the magnetic field lines are non-changing.

Model $\mathrm{b}$ is a hydrodynamical model showing the forward shock emission of a source with a random magnetic field generated by shock instabilities (e.g. [32]). If this source was viewed along the jet axis then no measurement of polarisation would be made as the magnetic field geometry would cause the signal to cancel. However, a polarisation signal can be detected from a source with a random magnetic field if the line of sight to the GRB runs along the edge of the jet cone. This is possible when the shock instability produces anisotropic random magnetic fields (e.g. the component parallel to the shock front has a higher value). In such a case each fluid element produces polarised emission. If the viewing angle is close to the edge of the jet cone, the observable region around the line of sight is asymmetric and the net polarisation is not cancelled out. For this model it is predicted that there would be a jet break (i.e. a steepening of the light curve) coincident with a flip in the polarisation angle as the observable area of the jet increases and becomes larger than the jet itself causing the symmetry to be restored by the beamed emission from the other side of the jet. Model $b$ can also be applicable to the reverse shock emission if the original ejecta does not contain large scale magnetic fields.

Model $\mathrm{c}$ is a hydrodynamical model showing the forward shock emission of a source with coherent patches of magnetic field. Each patch generates the maximum polarisation of $\Pi_{0} \sim 70 \%$ but the maximum total polarisation is reduced according to the number of patches making up the jet, i.e. $\Pi_{0} / \sqrt{\# \text { patches }} \sim 10 \%$. This model predicts fluctuating polarisation angle measurements due to the patches themselves having fluctuating angles [28]. Model c can also be applicable to the reverse shock emission if the original ejecta doesn not contain large scale magnetic fields.

To enhance our ability to distinguish between theoretical light curves and jet models and place tighter constraints on the structure of the magnetic field within the GRB jets, we need to be able to explore the temporal and colour evolution of the polarisation. This advance in understanding is

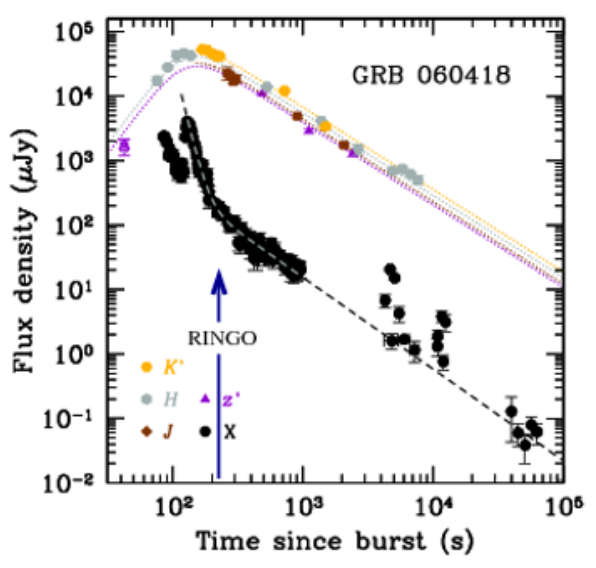

Figure 4. Observed light curve for GRB 060418 with arrow indicating RINGO measurement [35].

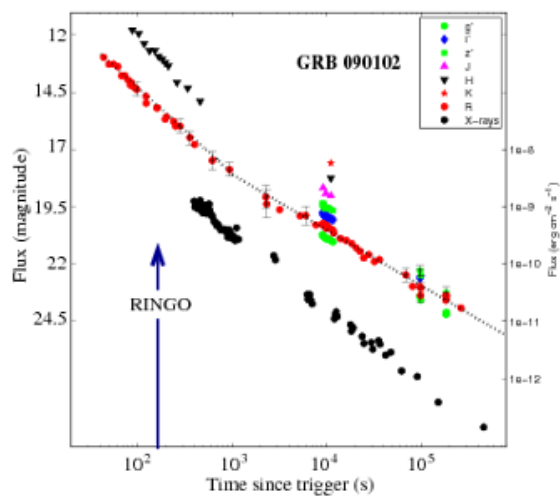

Figure 5. Observed light curve for GRB 090102 with arrow indicating RINGO measurement [38].

possible with the advent of the second and third generation RINGO polarimeters on the LT.

\section{Results and Discussion}

\subsection{GRB 060418}

The RINGO polarimeter monitored its first GRB in April 2006: GRB 060418. The source was detected by the SWIFT satellite [34] and triggered observations at the LT $\sim 200$ seconds after the outburst. The result was a $2 \sigma$ upper limit on optical polarisation of $\mathrm{P}<8 \%$ [35]. This measurement coincided with the deceleration of the fireball and the onset of the afterglow emission ([35]) which is made up of an unknown contribution from the forward and reverse shocks. The light curve for GRB 060418 (see Figure 4) is most likely to correspond with a type III light curve model (see Figure 2) due to the fact that the afterglow decays as $\mathrm{t}^{-1}$ [36] (rather than $\mathrm{t}^{-2}$ for reverse shock [37]).

The forward shock dominates the emission in the type III light curve (see Figure 2), however it is not known what percentage of the emission comes from the reverse 
shock. A simple, standard model predicts that the forward shock and reverse shock emission equally contribute to the early afterglow at the onset. We are able to say whether or not each theoretical model is consistent with observations, however, the interpretation is not unique. It is very unlikely that GRB 060418 has an off axis disordered magnetic field model due to the absence of a jet break in the light curve. Without knowing how much the reverse shock contributes to the emission it is not possible to distinguish between models a and $\mathrm{c}$ in Figure 3. It is possible that the forward and reverse shock contribute an equal number of photons to the emission which would mean there is no ordered magnetic field in the jet (model c), alternatively it is possible that the forward shock contributed more than half the photons to the emission and the low polarisation measurement is due to this dominance and does not rule out the presence of a large-scale ordered magnetic field. For these reasons it is not possible to make a definitive statement about the magnetic field structure in GRB 060418.

\subsection{GRB 090102}

GRB 090102 was monitored by the RINGO polarimeter in January 2009 ([38]). A 60 second exposure was taken just 160 seconds after the trigger time (the first detection of optical polarisation of a GRB afterglow at early time) and detected $P=10.2 \pm 1.3 \%$. The shape of the light curve (see Figure 5) corresponds with the type II light curve model (see Figure 2) whereby the reverse shock emission decays and a flattening occurs representative of the forward shock emission. The RINGO polarisation measurement was coincident with the reverse shock phase of the light curve (see Figure 5), the detection of $\sim 10 \%$ polarisation is consistent with the magnetised model in which large-scale magnetic fields are produced at the central engine.

This measurement makes it unlikely that the jet of GRB 090102 is made up of coherent patches of magnetic field. As described in Section 3 (see model c in Figure 3) if the jet structure was that of coherent patches of ordered magnetic field the optimistic upper limit for the degree of polarisation would be $\sim 10 \%$. With the RINGO measurement of $\mathrm{P}=10.2 \pm 1.3 \%$ (over a period of 60 seconds) at the top end of this limit it is unlikely that GRB 090102 corresponds with a coherent patches model.

The shape of the light curve eliminates the possibility that the magnetic field structure in the jet of GRB 090102 is a random magnetic field with a viewing angle along the optical axis (see image $b$ in Figure 3 ). This model predicts a steepening (a 'jet break') of the light curve coincident with the observable region of the jet expanding to a size larger than the jet itself. The light curve for GRB 090102 in Figure 5 shows an observed flattening of the curve that corresponds with the transition from the reverse to forward shock dominating the light curve. With no jet break in the observed light curve it is unlikely that GRB 090102 has a disordered magnetic field.

The uniform magnetic field model (see model a in Figure 3 ) predicts a constant polarisation angle and a decrease in polarisation percentage with time. This is the most likely model for the structure of the magnetic field in the jet of GRB 090102 as the RINGO measurement corresponds with the predictions for a jet with an ordered magnetic field. However, in order to place tighter constraints on the GRB jet structure it is imperative that measurements of the polarisation degree and angle are taken temporally as the afterglow fades. Combining these temporally and spectrally evolving values with the observed optical light curve will be a powerful method in exploring the magnetic field structure in GRBs.

\section{Future Work}

The introduction of the RINGO polarimeters to the LT has opened up the possibility to explore the structure of the magnetic field in the jets of GRBs. The second and third generation RINGO polarimeters afford us the ability to monitor the temporal (RINGO2 \& RINGO3) and colour (RINGO3) evolution of the polarisation which will allow us to place tighter constraints on jet magnetisation and the structure of the magnetic field. In 2009 RINGO2 was decommisioned and enhanced to make RINGO3 which is currently actively responding to GRB triggers, there are a wealth of data from RINGO2 which is being analysed by the Liverpool GRB Team and soon to be published.

The RINGO polarimeters are also instrumental in the regular and intensive monitoring of blazars which started as an extension of the photometric Tuorla blazar monitoring program [39]. The LT regularly monitors a sample ${ }^{1}$ of 16 AGN, (13 of which are blazar sources) which have gone into flaring mode over the last $\sim 3$ years. The main focus of the project is to combine optical and gamma-ray astronomy to untangle the sequence of events leading to gamma-ray flares in blazars. The optical polarisation data are combined with data from the MAGIC [40] large atmospheric imaging telescope and the F-GAMMA (FERMIGST AGN Multi-frequency Monitoring Alliance) group [41] who collaborate with the LT team to combine optical polarimetry follow-up with gamma-ray data to better understand the physics behind the $\mathrm{TeV}$ flares and jets.

The LT blazar sample includes some of the most interesting blazars such as OJ287, Markarian 421 and BL Lac (see Table 1), along with the nearby radio galaxy source M87 (see reference [42]). The blazars are regularly monitored by the LT, MAGIC and FERMI in order to follow the variability of the source over long time periods and also intensively monitored during periods of activity to probe the intra-night variability of the sources. The blazar team currently have RINGO2 \& RINGO3 data for all the sources in the sample (see Table 1) including data from an intensive observational campaign of Markarian 421 when it entered flaring mode this year. There are also results for $3 \mathrm{C} 279$ which are to be published soon. The polarisation data for the other sources in the sample are in the process of being reduced with the intention of publishing the initial results in early 2014.

${ }^{1}$ See http://www.users.utu.fi/kani/1m/index.html for light curves 


\begin{tabular}{lcccc}
\multicolumn{5}{c}{ LT BlazAR AND AGN SAMPLE } \\
\hline Object & Right Ascension & Declination & Redshift (z) & Type \\
\hline NGC 1275 & $03: 19: 48.10$ & $+41: 30: 41.9$ & 0.018 & Radio galaxy \\
Mrk 501 & $16: 53: 52.20$ & $+39: 45: 37.0$ & 0.034 & BL Lac \\
Mrk 421 & $11: 04: 27.30$ & $+38: 12: 32.0$ & 0.031 & BL Lac \\
Mrk 180 & $11: 36: 26.40$ & $+70: 09: 27.0$ & 0.045 & BL Lac \\
IC 310 & $03: 16: 43.00$ & $+41: 19: 30.0$ & 0.019 & Radio galaxy \\
3C 279 & $12: 56: 11.17$ & $-05: 47: 21.5$ & 0.536 & FSRQ \\
PKS 1222+216 & $12: 24: 54.40$ & $+21: 22: 46.0$ & 0.432 & FSRQ \\
S5 0716+714 & $07: 21: 53.45$ & $+71: 20: 36.4$ & 0.31 & BL Lac \\
3C 66A & $02: 22: 39.61$ & $+43: 02: 07.8$ & 0.444 & BL Lac \\
1ES 1011+496 & $10: 15: 04.19$ & $+49: 26: 01.0$ & 0.212 & BL Lac \\
M87 & $12: 30: 49.40$ & $+12: 23: 28.0$ & 0.004 & Radio galaxy \\
BL Lac & $22: 02: 43.28$ & $+42: 16: 40.0$ & 0.069 & BL Lac \\
ON 231 & $12: 21: 31.69$ & $+28: 13: 58.5$ & 0.102 & BL Lac \\
OJ 287 $87: 54: 48.90$ & $+20: 06: 30.9$ & 0.306 & BL Lac \\
PG 1553+113 & $15: 55: 43.00$ & $+11: 11: 24.0$ & $<0.78$ & BL Lac \\
PKS 1510-089 & $15: 12: 50.53$ & $-09: 05: 59.8$ & 0.360 & FSRQ \\
\hline \hline
\end{tabular}

Table 1. The AGN sample monitored by the LT. The table shows the sources' co-ordinates (in the J2000 equatorial system), redshift and galaxy type. The sources that are not strictly blazars are kept in the sample for interest, particularly due to their possible status as orientated blazar-like AGN and also their close proximity which allows a closer exploration of jet structure (in the case of M87 [42]).

\section{References}

[1] R. D. Blandford \& D. G. Payne, MNRAS, 199, 883903, (1982)

[2] T. Piran, RvMP., 76, 1143-1210, (2004)

[3] P. Meszaros, Reports on Progress in Physics, 69, 2259-2321, (2006)

[4] T. Piran, ApJ, 389, L45-L48, (1992)

[5] M. Lacy et al., ApJ, 551, 17-21, (2001)

[6] R. D. Blandford \& R. L. Znajek, MNRAS, 179, 433456. (1977)

[7] T. Hovatta et al., A\&A, 494, 527-537 (2009)

[8] M. Ackermann et al., ApJ, 716, 1178-1190, (2010)

[9] R. S. Nemmen et al., Science, 338, 1445-1448, (2012)

[10] R. Antonucci, ARA\&A, 31, 473-521 (1993)

[11] C. M. Urry \& P. Padovani, PASP, 107, 803-846 (1995)

[12] A. de Ugarte Postigo et al., A\&A, 462, L57-L60, (2007)

[13] Z. Cano et al., ApJ, 740, 41-57, (2012)

[14] D. Q. Lamb, T. Q. Donaghy \& C. Graziani, ApJ, 620, 355-378, (2005)

[15] I. A. Steele et al., SPIE, 5489, 679 (2004)

[16] P. Roche \& N. Szymanek, IAU Commission, 55, 384-386, (2005)

[17] S. D. Barthelmy, SPIE, 4140, 50-63, (2000)

[18] N. Gehrels et al., ApJ, 611, 1005-1020 (2004)

[19] C. Guidorzi et al., PASP, 118, 288-296 (2006)

[20] I. A. Steele et al., SPIE 6269, 179-192 (2006)

[21] D. Clarke \& D. Neumayer, 383, 360-366 (2002)

[22] D. M. Arnold et al., SPIE, 8446, 2-10 (2012)
[23] I. A. Steele et al., SPIE, 7735, 142-154 (2010)

[24] S. Kobayashi, T. Piran \& R. Sari, ApJ, 513, 669-678, (1999)

[25] C. G. Mundell, C. Guidorzi \& I. A. Steele, Advances in Astronomy, 718468, 1-20 (2010)

[26] B. Zhang \& S. Kobayashi, ApJ, 628, 315-334 (2005)

[27] Y. Z Fan, D. M. Wei, C. F. Wang, A\&A, 424, 477484 (2004)

[28] A. Gruzinov \& E. Waxman, ApJ, 511, 852-861, (1999)

[29] G. Drenkhahn \& H. C. Spruit, A\&A, 391, 11411153, (2002)

[30] M. Lyutikov, V. I. Pariew \& R. D. Blandford, ApJ, 597, 998-1009, (2003)

[31] B. Zhang, S. Kobayashi, P. Meszaros, ApJ, 595, 950954, (2003)

[32] G. Ghisellini \& D. Lazzati, MNRAS, 309, L7-L11, (1999)

[33] M. Lyutikov, New Journal of Physics, 8, 119-143, (2006)

[34] A. D. Falcone et al., GCN, 4966, 1, (2006)

[35] C. G. Mundell et al., Science, 315, 1822-1824 (2007)

[36] R. Sari, T. Piran \& R. Narayan, ApJ, 497, L17-L20, (1998)

[37] S. Kobayashi, ApJ, 545, 807-812, (2000)

[38] I. A. Steele et al., Nature, 462, 767-769 (2009)

[39] L. O. Takalo et al., 1085, 705-707, (2008)

[40] U. Barres de Almeida, HEPRO III, 8, 178-183, (2012)

[41] A. A. Abdo et al., ApJ, 700, 597-622, (2010)

[42] A. Abramowski et al., 746, 151-169, (2012) 